\title{
Management of pemphigus vulgaris: challenges and solutions
}

This article was published in the following Dove Press journal:

Clinical, Cosmetic and Investigational Dermatology

2I October 2015

Number of times this article has been viewed

\section{Stamatis Gregoriou \\ Ourania Efthymiou \\ Christina Stefanaki \\ Dimitris Rigopoulos}

2nd Department of Dermatology and Venereology, University of Athens Medical School, Attikon Hospital, Athens, Greece
Correspondence: Stamatis Gregoriou 2nd Department of Dermatology Venereology, University of Athens Medical School, Attikon Hospital, I Rimini Street, Athens 12462, Greece Tel +302105832396

$\mathrm{Fax}+302105832397$

Email stamgreg@yahoo.gr
Abstract: The main objective in the treatment of pemphigus vulgaris is to control the disease, prevent relapses, and avoid adverse events associated with the prolonged use of steroids and immunosuppressive agents. Systemic corticosteroids remain the gold standard treatment for pemphigus vulgaris. Azathioprine and mycophenolate mofetil are the first line of steroid-sparing treatment. Rituximab is extremely effective in recalcitrant pemphigus, when other treatments fail to control the disease. The European Dermatology Forum recommends tapering prednisolone by $25 \%$ every 2 weeks after the consolidation phase, and a $5 \mathrm{mg}$ reduction every 4 weeks when the dose is reduced to $<20 \mathrm{mg}$. If the patient relapses, options include increasing steroids back to the previous dose, adding an immunosuppressant if using steroid monotherapy, or replacing a first-line immunosuppressant by another if already on combination therapy.

Keywords: pemphigus vulgaris, treatment, steroids, rituximab, azathioprine, mycophenolate

\section{Pemphigus vulgaris: treatment challenges}

Pemphigus vulgaris (PV) is a potentially fatal autoimmune, intraepithelial disease characterized by flaccid blisters and erosions of the skin and mucous membranes and histologically by acantholysis. ${ }^{1}$ It is mediated by circulating desmoglein-reactive autoantibodies directed against keratinocyte cell surfaces. ${ }^{2}$

The primary objective of the therapeutic management of PV is initially to control the disease, heal the bullous skin and mucous lesions, and minimize the associated functional impairment. Subsequently, the real challenge is to prevent relapses in the long run and avoid adverse events associated with the prolonged use of steroids and immunosuppressive agents. Such intent requires close clinical monitoring of efficacy and safety of treatment. ${ }^{3}$ The rapid tapering of steroids once control of the disease has been achieved and the introduction of novel immunosuppressants with less long-term adverse events have decreased both morbidity and mortality of pemphigus patients in the last decades. ${ }^{4}$ However, there are still unmet needs in pemphigus therapy. Even though medical literature highlights the pivotal significance of tapering the immunosuppressive treatment, there are few guidelines on the duration of maintenance therapy or the prerequisites of discontinuation. The objective should be to achieve as rapid a remission as possible, as few flare-ups as possible, and minimal morbidity associated with treatment agents. The challenge is to minimize hospitalization and improve patients' quality of life.

$\mathrm{PV}$ is characterized by diversity that makes every patient a unique challenge. Patients might present with lesions solely on mucous membranes and have limited cutaneous or extensive mucocutaneous involvement. Comorbidities such as diabetes mellitus, hypertension, previous or existent malignancies, chronic infections, and associated 
complications might limit available treatment options. In addition, treating patients during the onset of the disease is quite different than treating patients with recalcitrant disease or patients after multiple remissions, since the latter usually have more compromised health-related quality of life and compliance with treatment. This diversity in patient population along with the rarity of the disease is the main reason for the limited number of comparative studies in pemphigus treatment. There are many therapeutic interventions in use that have not been evaluated in well-designed randomized controlled trials (RCTs). Sample sizes of conducted trials are often small and have been occasionally evaluated as insufficient to yield definitive results. ${ }^{5}$ Most published data regard patients during disease onset. Duration of follow-up also varies in the literature, limiting the capacity to conduct long-term risk-benefit analysis. Lack of uniform outcome measures limits the possibility of meta-analysis and the strength of evidence-based treatment guidelines. An effort has been made to introduce common definitions and end points in PV treatment by the European Dermatology Forum $(\mathrm{EDF})^{3}$ and a recent international consensus. ${ }^{6}$ In addition, development of severity scores for the disease will help in comparative evaluation of different trials. Evaluation of mild, moderate, or severe disease differs among experts. Some authors define severity on the basis of body surface area affected, while others use the number of new blisters per day to delineate between limited and severe disease. ${ }^{7-9}$ However, even in the absence of new blisters and regardless of affected areas, the involvement of functional critical sites such as mucosal surfaces or ocular disease may require more aggressive therapy.

Common definitions, end points, and severity scores mean that even data from underpowered trials can be used in conjunction with other similar ones in a meta-analysis. The purpose of this review is not to provide a detailed evaluation of all treatment options in PV. We will focus on controversial issues in the use of the most common agents and how to stand up to the treatment challenges based on literature evidence when available.

\section{Consensus statement}

The consensus statement of mutually acceptable common definitions for pemphigus was developed by the International Pemphigus Committee. ${ }^{6}$

Baseline is defined as the day that therapy is started by a physician.

Control of disease activity is the time from baseline to the time at which new lesions cease to form and established lesions begin to heal. This marks the beginning of the consolidation phase.

End of consolidation phase is defined as the time at which no new lesions have developed for a minimum of 2 weeks and approximately $80 \%$ of established lesions have healed. Clinicians start to taper corticosteroids (CSs).

Complete remission on therapy is defined as the absence of new or established lesions for at least 2 months while the patient is receiving minimal therapy.

Minimal therapy is defined as $\leq 10 \mathrm{mg} / \mathrm{d}$ prednisolone (or equivalent) and/or minimal adjuvant therapy for at least 2 months.

Minimal adjuvant therapy is defined as half of the dose required to be defined as treatment failure.

Complete remission off therapy is defined as the absence of new and/or established lesions for at least 2 months while the patient is off all systemic therapy for at least 2 months.

Relapse/flare is the appearance of $\geq 3$ new lesions/month that do not heal spontaneously within 1 week, or by the extension of established lesions, in a patient who has achieved control of disease activity.

One could argue that such definitions are more suitable in a clinical study environment. However, they can easily be implemented even in everyday hospital clinical practice without complicated calculations, and offer an invaluable common evaluation of each patient in every treatment stage.

\section{Treatment agents}

The aim of pharmacologic therapy for PV is to reduce inflammatory response and autoantibody production.

\section{Steroids}

Systemic CSs are still the first-line treatment for PV. One of the main concerns in uncomplicated patients is when rapid control of the disease is achieved by monotherapy with CSs. Control of disease activity is usually achieved within several weeks. Complete remission on minimal treatment needs months, while complete remission off treatment often requires several months or even years of therapy. ${ }^{10}$

A second debate often concerns whether to start with a low or high dose of CSs. The guidelines by EDF and European Academy of Dermatology and Venereology recommend initial prednisolone dose at $0.5 \mathrm{mg}-1.5 \mathrm{mg} / \mathrm{kg} / \mathrm{d}$ and if control of the disease is not reached within 2 weeks, a higher prednisolone dose (up to $2 \mathrm{mg} / \mathrm{kg}$ ) could be administered. ${ }^{3}$ The optimal dose has not been validated. A controlled trial showed no significant difference regarding the duration of remissions and 
relapse rates at 5 years in patients randomized to treatment with either low-dose oral prednisolone $(1 \mathrm{mg} / \mathrm{kg} / \mathrm{d})$ or highdose oral prednisolone (2.0-2.5 mg/kg/d). ${ }^{11}$ Once remission is induced and maintained with healing of the majority of lesions, the dose can be tapered by $25 \%$. Reduction may be performed biweekly with slower decreases when doses below $20 \mathrm{mg} / \mathrm{d}$ are reached. ${ }^{12}$

CSs can be combined with an immunosuppressive agent, particularly when complications due to expected prolonged use ( $>4$ months) such as hypertension, diabetes mellitus, and osteoporosis are expected. ${ }^{3}$ Even though the superiority of steroids plus adjuvant therapy over prednisolone monotherapy is debatable, considerable research effort has been directed at finding the optimal steroid-sparing agent. ${ }^{13,14}$ A recent systematic review that evaluated RCTs with adjuvant therapy with azathioprine, mycophenolate mofetil (MMF), cyclophosphamide, cyclosporine, intravenous immunoglobulin (IVIG), plasma exchange, and infliximab in PV patients concluded that adjuvants were not beneficial for achieving remission, but were found to collectively decrease the risk of relapse by $29 \% .^{15}$

If doses of prednisolone above $100 \mathrm{mg} / \mathrm{d}$ are required, pulse treatment with either oral or intravenous (IV) steroids may be considered. A regimen of IV betamethasone in combination with oral prednisolone in PV patients showed a shorter time to remission, clinical resolution (including oral lesions), and minor adverse effects when compared to monotherapy with oral prednisolone. ${ }^{16} \mathrm{~A}$ common pulse regimen used is $100 \mathrm{mg} / \mathrm{d}$ of IV dexamethasone for 3 days every $2-3$ weeks. ${ }^{17}$ However, pulsed CSs do not appear to have additional benefit on top of conventional first-line treatment with oral prednisolone and immunosuppressive adjuvants. ${ }^{18}$ Most of the studies investigating the efficacy of pulse steroid treatment involve patients with refractory PV. In such patients, pulsed therapy with IV dexamethasone could be a reliable alternative when other options have failed.

Second-line treatment in the case of contraindications to glucocorticoids or complications due to expected prolonged use ( $>4$ months) consists in the combined or single use of immunosuppressants such as azathioprine, MMF, dapsone, methotrexate, cyclophosphamide, and cyclosporine. In recent years, the use of IVIG and biologics such as infliximab, and especially rituximab, has been reported to produce excellent results in refractory cases.

\section{Azathioprine}

Azathioprine is one of the main adjuvants used in PV. ${ }^{19}$ It is considered a first-line adjuvant immunosuppressant according to the EDF guidelines. Dose varies between 1 and $3 \mathrm{mg} / \mathrm{kg} / \mathrm{d}$, based on the activity of the thiopurine methyltransferase (TPMT) enzyme, involved in the metabolism of the drug. When TPMT levels are high, normal doses of azathioprine (up to $2.5 \mathrm{mg} / \mathrm{kg} / \mathrm{d}$ ) are administered, while adults with PV and intermediate or low TPMT levels should receive a maintenance dose (up to $0.5-1.5 \mathrm{mg} / \mathrm{kg} / \mathrm{d}$ ). Azathioprine should not be used in patients with no TPMT activity. A dose of $50 \mathrm{mg} / \mathrm{d}$ could initially be administered, and if no idiosyncratic reactions occur, it could be increased after a week. In case of idiosyncratic reactions, it should be discontinued. ${ }^{20-22}$ The primary benefit of adjuvant azathioprine is its steroid-sparing effect. ${ }^{23}$ Azathioprine has been reported to require a lower cumulative $\mathrm{CS}$ dose for remission, with some investigators reporting superior steroid-sparing effect when compared to MMF and cyclophosphamide, ${ }^{24}$ while others concluded that cyclophosphamide is superior. ${ }^{13}$ Adverse events of adjuvant azathioprine treatment are decreased when compared to steroid monotherapy without any compromise in rates of clinical remission. ${ }^{25}$

\section{Mycophenolate mofetil}

MMF is a safe steroid-sparing agent. ${ }^{26}$ It is considered a first-line adjuvant immunosuppressant according to the EDF guidelines. The optimal dose is weight dependent with a dose of $2 \mathrm{~g} / \mathrm{d}$ recommended for the average patient of 75 $\mathrm{kg}$. Progressive dose increase by $500 \mathrm{mg} /$ wk until the final dose of $2 \mathrm{~g} / \mathrm{d}$ has been proposed to avoid gastrointestinal adverse events. ${ }^{1}$ Efficacy is debated. In a recent RCT, MMF ( 2 or $3 \mathrm{~g} / \mathrm{d}$ ) plus oral CSs was not found to be superior when compared with oral CSs and placebo in patients with mild or moderate PV. The primary end point was patients responding to treatment. ${ }^{27}$ Other investigators have also reported no clinical benefit using adjuvant MMF to steroids in patients with $\mathrm{PV} .{ }^{28} \mathrm{MMF}$ in combination with prednisolone seems to have a more prominent beneficial role in patients with relapses of $\mathrm{PV}^{29}$ or in cases of refractory $\mathrm{PV}$ who have failed previous treatments. ${ }^{30}$

\section{Cyclophosphamide}

Cyclophosphamide is considered a second-line immunosuppressant adjuvant therapy according to the EDF guidelines. It can be administered either as a $500 \mathrm{mg}$ IV infusion or as $2 \mathrm{mg} / \mathrm{kg} / \mathrm{d}$ orally. ${ }^{3}$ Cyclophosphamide monotherapy has not been able to demonstrate any benefit over prednisolone. ${ }^{31}$ Some authors report superiority over azathioprine or mycophenolate as adjuvant therapy. ${ }^{13}$ The potential long-term side effects (infertility, increased risk of cancer, infections, 
genitourinary complications, and lymphopenia) further limit cyclophosphamide's use. ${ }^{32}$

\section{Dapsone}

Dapsone is recommended in a dose of $100 \mathrm{mg} / \mathrm{d}$ or up to $\leq 1.5 \mathrm{mg} / \mathrm{kg} / \mathrm{d}$ as a steroid-sparing agent. ${ }^{3}$ An RCT reported superiority of dapsone over placebo as a steroid-sparing agent when the primary end point was to taper prednisolone to $\leq 7.5 \mathrm{mg} / \mathrm{d}$. However, dapsone did not exhibit any benefit on remission of the disease. ${ }^{33}$ Before initiating therapy with dapsone, serum G6PD activity should be tested.

\section{Methotrexate}

Methotrexate could be used as a steroid-sparing agent in a dose of $10-20 \mathrm{mg} / \mathrm{wk} .{ }^{3}$ Literature data assessing its efficacy in $\mathrm{PV}$ treatment are scarce. A recent retrospective study reported that 21 out of 25 patients downgraded PV severity and were able to taper steroids after 6 months when using adjuvant therapy with $15 \mathrm{mg}$ of methotrexate per week. ${ }^{34}$

\section{Rituximab}

Rituximab is an anti-CD20 monoclonal humanized antibody with the potential to reduce desmoglein autoantibodies and selectively deplete B cells. ${ }^{35-37}$ Rituximab is indicated in patients who remain dependent on more than $10 \mathrm{mg}$ prednisolone combined with an immunosuppressive adjuvant according to the EDF. Administration schedule in literature is either 1,000 mg IV every 2 weeks or $375 \mathrm{mg} / \mathrm{m}^{2}$ every week..$^{38-44}$ The same dosage can be administered again in case of clinical relapses. A meta-analysis on treatment with rituximab in severe pemphigus showed remission in approximately $95 \%$ of the total patients. ${ }^{35}$ Prophylactic infusion after complete remission does not seem to provide any additional benefit. ${ }^{42}$ The incidence of serious infections was 3.9\% using the weekly protocol but $15.21 \%$ in the biweekly protocol. ${ }^{45}$ However, the incidence of unforeseen fatal infections such as progressive multifocal leukoencephalopathy cannot be estimated due to the rarity of the desease. ${ }^{3}$ Concomitant longterm antibiotic and prophylaxis for herpes virus has been shown to drastically reduce the rate of infections. ${ }^{42}$ Rituximab does not eliminate the need for steroids or immunosuppressive agents, and most patients in published studies did use such therapy along with rituximab. ${ }^{35}$ Before initiation of treatment, physicians should have a specific goal and end point. They should also be aware of its potential side effects and lack of information on its long-term effects. Patients should be carefully monitored during and after therapy. ${ }^{45}$

\section{Intravenous immunoglobulins}

Treatment with IVIG can be used in refractory disease or in case of contraindications to immunosuppressive adjuvants. The usual dose is $2 \mathrm{~g} / \mathrm{kg} /$ cycle IV administered over $2-5$ consecutive days, monthly. ${ }^{3}$ A multicenter RCT that compared various doses of IVIG and placebo infusion demonstrated the beneficial effect of IVIG in the management of refractory pemphigus, indicating a dose-response relationship of the treated patients. ${ }^{46}$ IVIG could be used as adjuvant therapy to systemic CSs and immunosuppressive adjuvants. ${ }^{47}$ Treatment should be performed over several days to avoid adverse effects such as headache and nausea. IVIG could induce aseptic meningitis in patients who commonly experience migraines, and is contraindicated in patients with complete IgA deficiency. ${ }^{48}$

\section{Infliximab}

Infliximab is a chimeric monoclonal antibody against tumor necrosis factor alpha (TNF- $\alpha$ ). TNF- $\alpha$ has been found to be strongly expressed by the acantholytic cells in $\mathrm{PV}^{49}$ There are several case reports and case series of PV patients successfully treated with infliximab. ${ }^{50,51}$ On the other hand, there are also several case series and a small comparative study showing no benefit in patients with PV treated with infliximab. ${ }^{52,53}$ There is also one case of a patient treated with infliximab for rheumatoid arthritis who developed pemphigus foliaceus. ${ }^{54}$ In the context of current evidence, infliximab does not have a role in the treatment of PV.

\section{Other therapeutic strategies}

In addition to the aforementioned agents, other therapeutic strategies for PV used by dermatologists worldwide in clinical practice include immunoadsorption, therapeutic plasma exchange - plasmapheresis - and extracorporeal photochemotherapy.

\section{Immunoadsorption}

Rapid removal of circulating autoantibodies against Dsg1 and Dsg3 can be achieved by immunoadsorption. It is indicated in patients with refractory PV when CSs combined with azathioprine or mycophenolate fail to control the disease. Four treatments of immunoadsorption on 4 consecutive days (2.5-fold plasma volume/d), repeated after 4 weeks, if needed, are the recommended schedule. ${ }^{3}$ Treatment could be undertaken in combination with immunosuppressive agents such as rituximab and cyclophosphamide. ${ }^{55,56}$ Contraindications include severe systemic infections, cardiovascular diseases, 
and hemorrhagic diathesis. While immunoadsorption is far superior to plasmapheresis in terms of efficacy and safety, the high cost of the adsorbers is the major limiting factor.

\section{Therapeutic plasma exchange - plasmapheresis}

Plasmapheresis is an extracorporeal blood purification technique, in which the blood is continuously removed from the patient and separated into cellular components and plasma; the cellular compartments are returned to the patients along with replacement fluidlike albumin. Plasma exchange has been described as an effective adjuvant therapy in severe PV patients in controlling disease activity by reducing serum levels of autoantibodies. ${ }^{57}$ Plasma exchange can be performed using a centrifugation device used in blood banks. The double filtration plasmapheresis is a newer procedure that currently prevails because of its safety advantage. ${ }^{58}$ In double filtration plasmapheresis, immunoglobulins are selectively removed, while the loss of albumin is minimized. There is no standardized protocol for the number and frequency of sessions; however, four or five plasma exchanges, each exchange consisting of 1-1.5 plasma volumes, over a period of 7-10 days constitute an adequate short-term therapy to remove $90 \%$ of the total initial body immunoglobulin burden. ${ }^{59}$

Plasma exchange is relatively safe, and the risk of infection associated with it is mainly due to the steroids and immunosuppressives given along with it. Other transient and minor adverse effects of plasma exchange that have been reported include thrombocytopenia, hypogammaglobulinemia, fluid overload leading to hypertension and pulmonary edema, hypoproteinemia, anemia, leucopenia, and hypocalcemia. ${ }^{60}$ Because of the rapid fluid shift occurring as a result of removal of proteins, which maintain the osmotic pressure, it can lead to severe problems in patients with compromised cardiac function.

\section{Extracorporeal photochemotherapy}

Extracorporeal photochemotherapy involves the collection of mononuclear cells with a cell separator, their irradiation with ultraviolet-A (UV-A) light in the presence of 8-methoxypsoralen, and reinfusion of the treated cells into the patient. The mechanism of action has not been fully illuminated. Current knowledge suggests that extracorporeal photochemotherapy is an amplifier of the immunogenicity of class I-associated peptides that are present on the surface of the collected mononuclear cells. ${ }^{61}$ It has been approved by the US FDA (Food and Drug Administration) for the treatment of cutaneous T-cell lymphoma, and encouraging results have been reported in the management of nonmalignant disorders of the immune system such as PV, scleroderma, systemic lupus erythematosus, rheumatoid arthritis, autoimmune diabetes mellitus, rejection of cardiac and renal allograft, and chronic graft versus host disease. There are a few case series of patients with PV treated of extracorporeal photochemotherapy, with most of the patients exhibiting significant clinical improvement and no adverse effects. ${ }^{62-64}$

\section{Step-by-step therapy after control of the disease}

The EDF proposed a useful treatment algorithm for use after the consolidation phase. The patients usually require 1-3 months for complete healing of the lesions.

- Start tapering steroids as soon as disease control is reached.

- Taper prednisolone by $25 \%$ every 2 weeks. When the patient reaches a dose $<20 \mathrm{mg}$, taper more slowly. A $5 \mathrm{mg}$ reduction every 4 weeks could be suitable for most of the patients.

- If less than 3 lesions reappear during tapering of oral CS therapy, raise dose to the last effective dose for the patient.

- If the patient presents with a relapse ( $>3$ lesions), reincrease oral CS therapy, going two steps back in the previous dose until control of the lesions is achieved. Subsequently, restart tapering of systemic steroids. If you cannot obtain disease control, go back to the initial dose.

- If oral CSs are given as monotherapy, add an immunosuppressant.

- If oral CSs are already combined with an immunosuppressant, consider replacing a first-line immunosuppressant by another or the use of a second-line immunosuppressant including immunoadsorption, IVIG, or rituximab.

- Monitor the patient for adverse events, and remember that prolonged immunosuppressive therapy increases the risk of side effects.

- If you can monitor titers of anti-DSg antibodies, remember that persistence of high levels of anti-Dsg1 has a positive predictive value for skin relapses, while the persistence of anti-Dsg3 IgG does not necessarily indicate a mucosal relapse.

\section{Conclusion}

CSs remain the gold standard treatment for PV. Finding the most effective steroid-sparing agent has formed much of the focus of recent research; however, evidence is inconclusive. 
Azathioprine and MMF are the first line of steroid-sparing treatment. Rituximab is extremely effective in recalcitrant PV where other treatments fail to control the disease. Systemic treatment for PV is effective and has managed to lower the incidence of adverse events and morbidity compared to the past. However, as more studies incorporate the common definitions and guidelines, the level of evidence and strength of therapy recommendation will improve, resulting in shorter treatment and better quality of life for the patients.

\section{Disclosure}

The authors report no conflicts of interest in this work.

\section{References}

1. Ioannides D, Lazaridou E, Rigopoulos D. Pemphigus. J Eur Acad Dermatol Venereol. 2008;22:1478-1496.

2. Amagai M, Klaus-Kovtun V, Stanley JR. Autoantibodies against a novel epithelial cadherin in pemphigus vulgaris, a disease of cell adhesion. Cell. 1991;67:869-877.

3. Hertl M, Jedlickova H, Karpati S, et al. Pemphigus. S2 guideline for diagnosis and treatment - guided by the European Dermatology Forum (EDF) in cooperation with the European Academy of Dermatology and Venereology (EADV). J Eur Acad Dermatol Venereol. 2015;29: 405-414.

4. Bystryn JC, Steinman NM. The adjuvant therapy of pemphigus. An update. Arch Dermatol. 1996;132:203-212.

5. Martin LK, Werth VP, Villaneuva EV, Murrell DF. A systematic review of randomized controlled trials for pemphigus vulgaris and pemphigus foliaceus. J Am Acad Dermatol. 2011;64:903-908.

6. Murrell DF, Dick S, Ahmed AR, et al. Consensus statement on definitions of disease end points and therapeutic response for pemphigus. J Am Acad Dermatol. 2008;58:1043-1046.

7. Pfütze M, Niedermeier A, Hertl M, Eming R. Introducing a novel autoimmune bullous skin disorder intensity score (ABSIS) in pemphigus. Eur J Dermatol. 2007;17:4-11.

8. Rosenbach M, Murrell DF, Bystryn JC, et al. Reliability and convergent validity of two outcome instruments for pemphigus. J Invest Dermatol. 2009;129:2404-2410.

9. Rahbar Z, Daneshpazhooh M, Mirshams-Shahshahani M, et al. Pemphigus disease activity measurments pemphigus disease area index, autoimmune bullous skin disorder intensity score, and pemphigus vulgaris activity score. J Am Acad Dermatol. 2014;150(3):266-272.

10. Almugairen N, Hospital V, Bedane C, et al. Assessment of the rate of long-term complete remission off therapy in patients with pemphigus treated with different regimens including medium- and high-dose corticosteroids. J Am Acad Dermatol. 2013;69(4):583-588. doi: 10.1016/j.jaad.2013.05.016.

11. Ratnam KV, Phay KL, Tan CK. Pemphigus therapy with oral prednisolone regimens: a 5-year study. Int J Dermatol. 1990;29:363-367.

12. Harman KE, Albert S, Black MM, British Association of Dermatologists. Guidelines for the management of pemphigus vulgaris. Br J Dermatol. 2003;149:926-937.

13. Olszewska M, Kolacinska-Strasz Z, Sulej J, et al. Efficacy and safety of cyclophosphamide, azathioprine, and cyclosporine (ciclosporin) as adjuvant drugs in pemphigus vulgaris. Am J Clin Dermatol. 2007;8(2): $85-92$.

14. Chams-Davatchi C, Esmaili N, Daneshpazhooh M, et al. Randomized controlled open-label trial of four treatment regimens for pemphigus vulgaris. J Am Acad Dermatol. 2007;57(4):622-628.

15. Atzmony L, Hodak E, Leshem YA, et al. The role of adjuvant therapy in pemphigus: a systematic review and meta-analysis. J Am Acad Dermatol. 2015;73(2):264-271.
16. Femiano F, Gombos F, Scully C. Pemphigus vulgaris with oral involvement: evaluation of two different systemic corticosteroid therapeutic protocols. J Eur Acad Dermatol Venereol. 2002;16: 353-356.

17. Fernandes NC, Menezes M. Pulse therapy in pemphigus: report of 11 cases. An Bras Dermatol. 2013;88(4):672-675.

18. Mentink LF, Mackenzie MW, Toth GG, et al. Randomized controlled trial of adjuvant oral dexamethasone pulse therapy in pemphigus vulgaris: PEMPULS trial. Arch Dermatol. 2006;142:570-576.

19. Schiavo AL, Puca RV, Ruocco V, et al. Adjuvant drugs in autoimmune bullous diseases, efficacy versus safety: facts and controversies. Clin Dermatol. 2010;28:337-343.

20. Anstey AV, Wakelin S, Reynolds NJ. British Association of Dermatologists Therapy, Guidelines and Audit Subcommittee. Guidelines for prescribing azathioprine in dermatology. Br J Dermatol. 2004;151: 1123-1132.

21. Badalamenti SA, Kerdel FA. Azathioprine. In: Wolverton SE, editor. Comprehensive Dermatologic Drug Therapy. 2nd ed. London: Elsevier; 2007:183-195.

22. Meggitt SJ, Anstey AV, Mohd Mustapa MF, Reynolds NJ, Wakelin S. British Association of Dermatologists' guidelines for the safe and effective prescribing of azathioprine 2011. Br J Dermatol. 2011;165: 711-734.

23. Chams-Davatchi C, Mortazavizadeh A, Daneshpazhooh M, et al. Randomized double blind trial of prednisolone and azathioprine, vs Prednisolone and placebo, in the treatment of pemphigus vulgaris. J Eur Acad Dermatol Venereol. 2013;27(10):1285-1292.

24. Chams-Davatchi C, Esmaili N, Daneshpazhooh M, et al. Randomized controlled open-label trial of four treatment regimens for pemphigus vulgaris. Arch Dermatol. 2007;57:622-628.

25. Chaidemenos G, Apalla Z, Koussidou T, et al. High dose oral prednisone vs prednisone plus azathioprine for the treatment of oral pemphigus: a retrospective, bi-centre, comparative study. J Eur Acad Dermatol Venereol. 2011;25:206-210.

26. Mimouni D, Anhalt GJ, Cummins DL, et al. Treatment of pemphigus vulgaris and pemphigus foliaceus with mycophenolate mofetil. Arch Dermatol. 2003;139:739-742.

27. Beissert S, Mimouni D, Kanwar AJ, et al. Treating pemphigus vulgaris with prednisone and mycophenolate mofetil: a multicenter, randomized, placebo-controlled trial. J Invest Dermatol. 2010;130: 2041-2048

28. Ioannides D, Apalla Z, Lazaridou E, Rigopoulos D. Evaluation of mycophenolate mofetil as a steroid-sparing agent in pemphigus: a randomized, prospective study. J Eur Acad Dermatol Venereol. 2012;26:855-860

29. Enk AH, Knop J. Mycophenolate is effective in the treatment of pemphigus vulgaris. Arch Dermatol. 1999;135:54-56.

30. Powell AM, Albert S, Al Fares S, et al. An evaluation of the usefulness of mycophenolate mofetil in pemphigus. Br J Dermatol. 2003;149: 138-145.

31. Chrysomallis F, Ioannides D, Teknetzis A, et al. Treatment of oral pemphigus vulgaris. Int J Dermatol. 1994;33:803-807.

32. Cummins DL, Mimouni D, Anhalt GJ, et al. Oral cyclophosphamide for treatment of pemphigus vulgaris and foliaceus. J Am Acad Dermatol. 2003;49:276-280.

33. Werth VP, Fivenson D, Pandya AG, et al. Multicenter randomized, double-blind, placebo-controlled, clinical trial of dapsone as a glucocorticoid-sparing agent in maintenance-phase pemphigus vulgaris. Arch Dermatol. 2008;144:25-32.

34. Baum S, Greenberger S, Samuelov L, et al. Methotrexate is an effective and safe adjuvant therapy for pemphigus vulgaris. Eur $J$ Dermatol. 2012;22:83-87.

35. Schmidt E, Goebeler M, Zillikens D. Rituximab in severe pemphigus. Ann NY Acad Sci. 2009;1173:683-691.

36. Zambruno G, Borradori L. Rituximab immunotherapy in pemphigus: therapeutic effects beyond B-cell depletion. J Invest Dermatol. 2008;128: $2745-2747$. 
37. Eming R, Nagel A, Wolff-Franke S, et al. Rituximab exerts a dual effect in pemphigus vulgaris. J Invest Dermatol. 2008;128:2850-2858.

38. Kasperkiewicz M, Shimanovich I, Meier M, et al. Treatment of severe pemphigus with a combination of immunoadsorption, rituximab, pulsed dexamethasone and azathioprine/mycophenolate mofetil: a pilot study of 23 patients. Br J Dermatol. 2012;166:154-160.

39. Hertl M, Zillikens D, Borradori L, et al. Recommendations for the use of rituximab (anti-CD20 antibody) in the treatment of autoimmune bullous skin diseases. J Dtsch Dermatol Ges. 2008;6:366-373.

40. Kanwar AJ, Vinay K, Sawatkar GU, et al. Clinical and immunological outcomes of high and low dose rituximab treatments in pemphigus patients: a randomized comparative observer blinded study. $\mathrm{Br} J$ Dermatol. 2014;170(6):1341-1349.

41. Amber K, Hertl M. An assessment of treatment history and its association with clinical outcomes and relapse in 155 pemphigus patients with response to a single cycle of rituximab. J Eur Acad Dermatol Venereol. 2015;29(4):777-782.

42. Gregoriou S, Giatrakou S, Theodoropoulos K, et al. Pilot study of 19 patients with severe pemphigus: prophylactic treatment with rituximab does not appear to be beneficial. Dermatology. 2014;228:158-165.

43. Lupi F, Masini C, Corona R, Puddu P, De Pità O: Therapy with rituximab for autoimmune pemphigus: results from a single-center observational study on 42 cases with long-term follow-up. J Am Acad Dermatol. 2012;67(4):617-622.

44. Ahmed AR, Spigelman Z, Cavacini LA, et al. Treatment of pemphigus vulgaris with rituximab and intravenous immune globulin. $N$ Engl $J$ Med. 2006;355:1772-1779.

45. Zakka LR, Shetty SS, Ahmed AR. Rituximab in the treatment of pemphigus vulgaris. Dermatol Ther (Heidelb). 2012;2:17.

46. Amagai M, Ikeda S, Shimizu H, et al, Pemphigus Study Group. A randomized double-blind trial of intravenous immunoglobulin for pemphigus. J Am Acad Dermatol. 2009;60:595-603.

47. Arnold DF, Burton J, Shine B, et al. An "n-of-1" placebo-controlled crossover trial of intravenous immunoglobulin as adjuvant therapy in refractory pemphigus vulgaris. Br J Dermatol. 2009;160:1098-1102.

48. Carbone J. Adverse reactions and pathogen safety of intravenous immunoglobulin. Curr Drug Saf. 2007;2:9-18.

49. López-Robles E, Avalos-Díaz E, Vega-Memije E, et al. TNFalpha and IL- 6 are mediators in the blistering process of pemphigus. Int $J$ Dermatol. 2001;40:185-188.

50. Jacobi A, Shuler G, Hertl M. Rapid control of therapy-refractory pemphigus vulgaris by treatment with the tumour necrosis factor-alpha inhibitor infliximab. Br J Dermatol. 2005;153:448-449.
51. Pardo J, Mercader P, Mahiques L, Sanchez-Carazo JL, Oliver V, Fortea JM. Infliximab in the management of severe pemphigus vulgaris. Br J Dermatol. 2005;153:222-223.

52. García-Rabasco A, Alsina-Gibert M, Pau-Charles I, Iranzo P. Infliximab therapy failure in two patients with pemphigus vulgaris. $J$ Am Acad Dermatol. 2012;67(5):e196-e197.

53. Hall RP 3rd, Fairley J, Woodley D, et al. A multicentre randomized trial of the treatment of patients with pemphigus vulgaris with infliximab and prednisone compared with prednisone alone. $\mathrm{Br} J$ Dermatol. 2015;172:760-768.

54. Boussemart L, Jacobelli S, Batteux F, et al. Autoimmune bullous skin diseases occurring under anti-tumor necrosis factor therapy: two case reports. Dermatology. 2010;221(3):201-205.

55. Zillikens D, Derfler K, Eming R, et al. Recommendations for the use of immunoapheresis in the treatment of autoimmune bullous diseases. J Dtsch Dermatol Ges. 2007;5:881-887.

56. Behzad M, M€obs C, Kneisel A, et al. Combined treatment with immunoadsorption and rituximab leads to fast and prolonged clinical remission in difficult-to-treat pemphigus vulgaris. $\mathrm{Br} J$ Dermatol. 2012;166:844-852.

57. Tan-Lim R, Bystryn JC. Effect of plasmapheresis therapy on circulating levels of pemphigus antibodies. J Am Acad Dermatol. 1990;22: $35-40$.

58. Kasuya A, Moriki M, Tatsuno K, Hirakawa S, Tokura Y. Clearance efficacy of autoantibodies in double filtration plasmapheresis for pemphigus foliaceus. Acta Derm Venereol. 2013;93(2):181-182.

59. Ranugha PS, Kumari R, Kartha LB, Parameswaran S, Thappa DM. Therapeutic plasma exchange as a crisis option in severe pemphigus vulgaris. Indian J Dermatol Venereol Leprol. 2012;78:508-510.

60. Yeh SW, Sami N, Ahmed RA. Treatment of pemphigus vulgaris: current and emerging options. Am J Clin Dermatol. 2005;6:327-342.

61. Perotti C, Torretta L, Viarengo G, et al. Feasibility and safety of a new technique of extracorporeal photochemotherapy: experience of 240 procedures. Haematologica. 1999;84:237-241.

62. Wollina U, Lange D, Looks A. Short-time extracorporeal 36. photochemotherapy in the treatment of drug-resistant autoimmune bullous diseases. Dermatology. 1999;198:140-144.

63. Gollnick HP, Owsianowski M, Taube KM, et al. Unresponsive severe generalized pemphigus vulgaris successfully controlled by extracorporeal photopheresis. J Am Acad Dermatol. 1993;28:122-124.

64. Prajapati V, Mydlarski PR. Advances in pemphigus therapy. Skin Therapy Lett. 2008;13:4-7.
Clinical, Cosmetic and Investigational Dermatology

\section{Publish your work in this journal}

Clinical, Cosmetic and Investigational Dermatology is an international, peer-reviewed, open access, online journal that focuses on the latest clinical and experimental research in all aspects of skin disease and cosmetic interventions. All areas of dermatology will be covered; contributions will be welcomed from all clinicians and

\section{Dovepress}

basic science researchers globally. This journal is indexed on CAS. The manuscript management system is completely online and includes a very quick and fair peer-review system, which is all easy to use. Visit http://www.dovepress.com/testimonials.php to read real quotes from published authors. 